\title{
Traduire
}

Revue française de la traduction

$237 \mid 2017$

La tête dans la toile

\section{Guide pratique pour traduire un site web}

\section{Aurélie Duclos}

\section{(2) OpenEdition \\ Journals}

Édition électronique

URL : http://journals.openedition.org/traduire/937

DOI : 10.4000/traduire.937

ISSN : 2272-9992

Éditeur

Société française des traducteurs

Édition imprimée

Date de publication : 1 décembre 2017

Pagination : 15-22

ISSN : 0395-773X

\section{Référence électronique}

Aurélie Duclos, « Guide pratique pour traduire un site web », Traduire [En ligne], 237 | 2017, mis en ligne le 01 décembre 2017, consulté le 29 juin 2019. URL : http://journals.openedition.org/traduire/937 ;

DOI : 10.4000/traduire.937 


\section{Guide pratique pour traduire un site web}

\section{Aurélie Duclos}

Pour de nombreux traducteurs, quel que soit leur domaine d'expertise, traduire pour le web est désormais une problématique incontournable. Toutefois, ils sont souvent désemparés voire légèrement paniqués face à une demande de traduction de site, ne sachant pas évaluer le temps de travail, avec un client néophyte qui attend du traducteur à la fois des conseils et une expertise. Je me permets donc de proposer ici un guide pratique visant à mieux appréhender le projet du client, grâce à un questionnaire bien ciblé. L'enjeu est à la fois de rassurer votre client, de proposer un devis adapté et de vous placer dans les meilleures conditions pour travailler sereinement.

\section{Distinguer les types de site web}

Avant toute chose, il faut commencer par cerner le type de site à traduire. Nous pouvons considérer qu'il en existe trois grands types :

- Les sites vitrines, qui sont une version un peu plus élaborée que la simple plaquette commerciale ou la carte de visite. Par exemple : le site entreprise de Danone.

- Les sites de commerce électronique, où l'on ajoute une base de données qui contient des produits à vendre. Par exemple : laredoute.fr

- Les services en ligne, qui sont des logiciels à part entière disponibles sur le web. Par exemple : Spotify ou Facebook.

À moins de vous spécialiser dans la localisation, vous n'aurez pas à travailler sur le troisième type de site dont les problématiques sont très spécifiques. Nous allons donc nous concentrer sur les deux premiers cas, qu'il est très simple de distinguer : s'il y a une fonction panier, c'est un site de commerce électronique, sinon c'est un site de présentation (avec peut-être quelques contenus dynamiques). 


\section{Identifier les contenus à traduire}

Vous allez, à ce stade, devoir déterminer ce qui doit être traduit, et c'est souvent la première difficulté que l'on rencontre. Si le client ne vous donne aucune information sur la manière d'extraire les contenus ou sur ce qu'il souhaite faire traduire dans son site, c'est souvent un signe que le projet manque de maturité. II est très probable que le client lui-même n'y a absolument pas réfléchi. Résultat : vous allez passer un temps considérable à faire des comptages pour votre devis, que ledit client mettra de côté parce qu'il "n'a pas le budget". Comptage qui, par ailleurs, sera approximatif et pourra s'avérer totalement caduc lorsque vous rentrerez dans le vif du sujet et découvrirez qu'il faut, en pratique, travailler avec une interface spécifique sur des contenus qui n'étaient pas visibles sur la partie publique du site.

II convient donc, à ce stade, de tout remettre à plat et de baliser avec le client les contenus qu'il souhaite faire traduire.

Vous pouvez commencer par hiérarchiser les contenus du site en fonction de leur rôle. On rencontre habituellement :

- des contenus statiques " classiques ": contenus des pages, mots-clés, architecture du site (menu, catégories), URL et métadonnées ;

- des contenus statiques juridiques : mentions légales, CGV, CGU ;

- des contenus dynamiques, tels que les pages générées à la demande, qui varient en fonction des informations saisies : compte client par exemple ;

- des fiches produit (site de commerce électronique) ;

- des contenus temporaires : offres promotionnelles, billets de blog, actualités de l'entreprise ; et/ou

- des contenus externes : e-mails automatiques et newsletters.

Vous pouvez déjà commencer à proposer des tarifs différenciés si vous souhaitez, par exemple, inclure la relecture des contenus juridiques confiée à une personne experte, et mettre de côté les fiches produit qui comporteront vraisemblablement beaucoup de répétitions (voir plus bas le cas du commerce électronique).

Au-delà de la fonction des contenus, il faudra sans doute évoquer leur nature. Vous rencontrerez du texte, mais un site peut aussi contenir :

- des images avec du texte ;

- des vidéos qui devront être sous-titrées ; et/ou

- des documents à télécharger. 
Faites un tour sur le site, sans le parcourir intégralement, pour voir si vous identifiez déjà certains de ces contenus. C'est à ce moment-là que vous allez prendre votre téléphone pour évoquer ces points un par un avec le client. Oui, oui, le téléphone, c'est nettement plus efficace et plus rapide!

\section{Parler à la bonne personne}

Lors de cette étape, il est important de comprendre qui est votre interlocuteur. Vous devrez évoquer des aspects à la fois budgétaires et techniques, potentiellement avec deux personnes différentes.

La personne responsable du budget et de la stratégie internationale est en général différente de l'expert technique. La première pourra vous indiquer ce qu'elle souhaite faire traduire (vous la guiderez avec les questions ci-dessus), la deuxième vous expliquera comment elle vous propose de le faire.

II ne servira à rien d'entrer dans des considérations techniques si vous parlez avec une personne qui a un rôle uniquement administratif ou managérial dans l'entreprise (PDG, secrétaire, responsable marketing, directeur des achats, etc.). Pour les aspects techniques, deux types de profil peuvent convenir :

- la personne qui alimente et administre le site au quotidien (webmaster) ; ou

- la personne qui a créé le site (développeur / intégrateur) - cette personne peut travailler en interne dans l'entreprise ou bien être un prestataire indépendant.

S'entretenir avec le responsable technique constitue une phase critique pour évoquer notamment le format des contenus et le processus de travail, informations qui seront à prendre en compte dans le devis. Deux options sont possibles : soit vous travaillez sur un export des contenus dans un ou plusieurs fichiers, soit directement sur le site, si celui-ci le permet. II est également possible de combiner les deux dans une approche hybride.

Remarque: les sites en ligne reposent pour la plupart sur un CMS (Content Management System - système de gestion de contenus), un outil qui fournit des "briques " que le développeur peut agencer en fonction de ses besoins. Les CMS les plus couramment rencontrés sont Wordpress, Joomla et Drupal pour les sites classiques, Prestashop et Magento pour les sites de commerce électronique. Lorsque vous travaillez sur une interface de traduction pour un client, c'est généralement sur un CMS. Dans certains cas, il peut s'agir d'un TMS (Translation Management System - système de gestion de la traduction), qui est un système tiers " raccordé " au site principal. Parmi les TMS connus sur le marché, on peut citer Transifex, Smartling, PhraseApp ou WebTranslatelt. 


\section{S'accorder sur une méthode de travail}

II n'existe pas dans l'absolu de méthode préférentielle : tout dépend de la nature des contenus à traduire, des délais, des contraintes du client, etc. Le tableau ci-dessous présente les avantages et inconvénients de chaque option pour le client :

\begin{tabular}{|l|l|l|}
\hline \multicolumn{1}{|c|}{ Avantages } & \multicolumn{1}{c|}{ Inconvénients } \\
\hline $\begin{array}{l}\text { Traduction } \\
\text { sur un fichier externe }\end{array}$ & $\begin{array}{l}\text { Compatible avec les outils d'aide à la } \\
\text { traduction (TAO) : IN-DIS-PEN-SA- } \\
\text { BLES pour les fiches produit du } \\
\text { commerce électronique. } \\
\text { Maîtrise du budget. }\end{array}$ & $\begin{array}{l}\text { Processus d'export et réintégration à } \\
\text { prévoir et à tester. }\end{array}$ \\
\hline $\begin{array}{l}\text { Traduction } \\
\text { sur un back-office web }\end{array}$ & Gain de temps sur l'intégration*. & $\begin{array}{l}\text { Risque de mauvaise manipulation du } \\
\text { contenu par le traducteur. } \\
\text { Formation sur l'outil à prévoir. }\end{array}$ \\
\hline
\end{tabular}

* Je n'ai pas mis " gain de coût ", car si le traducteur doit prendre en charge l'intégration en travaillant sur une plate-forme en ligne, les prix devront être adaptés en conséquence.

Sachant que les avantages et inconvénients ne sont pas vraiment les mêmes pour le traducteur :

\begin{tabular}{|l|l|l|}
\hline & \multicolumn{1}{|c|}{ Avantages } & \multicolumn{1}{c|}{ Inconvénients } \\
\hline $\begin{array}{l}\text { Traduction } \\
\text { sur un fichier externe }\end{array}$ & $\begin{array}{l}\text { Maîtrise du volume et des contenus. } \\
\text { Compatible TAO. }\end{array}$ & Formats potentiellement complexes. \\
\hline $\begin{array}{l}\text { Traduction } \\
\text { sur un back-office web }\end{array}$ & AUCUN & $\begin{array}{l}\text { Aucune maîtrise de la sauvegarde. } \\
\text { Risque de modification des contenus } \\
\text { après le devis. } \\
\text { Ralentissement dû à l'ergonomie. } \\
\text { Obligation de travailler en ligne (dépen- } \\
\text { dance vis-à-vis d'un réseau). }\end{array}$ \\
\hline
\end{tabular}

Cela ne veut pas dire qu'il faut absolument refuser de travailler en ligne, mais il faudra veiller à ce que le devis reflète le travail réel à fournir. II convient donc de peser le pour et le contre de chaque scénario avec le client. Par exemple, si le site compte dix pages, il est plus simple pour le client de les exporter une à une (même manuellement) et de les réintégrer en faisant un peu de mise en forme, que de former le traducteur à l'utilisation de son outil. Si le site compte en revanche 150 pages, il sera plus intéressant pour le client d'adopter la méthode 
inverse. Cela signifie pour le traducteur qu'il devra réussir à estimer le temps de travail nécessaire sur la plate-forme.

La règle d'or : ne jamais faire de devis pour une commande sur un back-office de traduction sans avoir pu tester l'outil en question. Si le client ne souhaite pas donner un droit d'accès (ce qui se défend, au stade du devis), il doit a minima transmettre des captures d'écran ou une vidéo de démonstration. Avec ces outils, il y a lieu de raisonner en temps passé : il sera en effet infiniment plus long de traduire 20 pages de 20 mots qu'une seule page de 400 mots, c'est pourquoi l'accès à l'outil en amont est primordial.

Par ailleurs, si le client vous " impose " de travailler en ligne, vous devrez lui soumettre vos propres exigences :

- il doit vous prévenir à l'avance de toute opération de maintenance pouvant avoir un impact sur votre travail ;

- il doit s'engager à ne pas modifier ni supprimer des contenus assignés ou en cours de traduction sans vous en avertir ; et

- il doit désigner un interlocuteur expert en interne sur l'outil, ou vous mettre en relation avec le prestataire qui l'administre.

Ces points sont à mentionner dans le devis pour vous protéger en cas de problème.

Conseil : même si vous optez pour une traduction en ligne, il vaut mieux exporter les blocs de texte qui dépassent les 500 mots au format HTML et travailler hors ligne pour vous protéger de tout " plantage " de l'outil et être en mesure d'utiliser votre logiciel de TAO. Attention au passage au logiciel Word, qui est à proscrire : un copier-coller depuis Word vers un outil en ligne crée en général de gros problèmes de mise en forme, car Word insère des balises qui lui sont propres dans le texte copié. Par ailleurs, toute la mise en forme de départ (titres, gras, italique) sera à reprendre au format HTML.

\section{Cas particulier des sites de commerce électronique}

La base de données produits représente souvent l'essentiel du contenu à traduire de ce genre de site. Le client a toujours la possibilité d'exporter avec un CMS un fichier au format .csv (soit un tableur au format texte, où chaque colonne est séparée par une virgule ou un point-virgule, et où chaque ligne représente un produit). Ce fichier pourra ensuite être traité avec un outil de TAO.

C'est là que vous pouvez aider votre client, si vous êtes un peu bricoleur, avec Excel ou avec votre outil de TAO : bien souvent, le fichier exporté est " brut " et parfois mal structuré. Faute de traitement du fichier, cela peut entraîner un coût de traduction élevé qui pourrait être largement optimisé. 
Prenons l'exemple d'un fichier .csv structuré comme ceci :

Identifiant produit ; description produit ; collection

102564 ; Pull col V - $100 \%$ coton, manches longues, bleu ciel ; été 2016

102565 ; Pull col V - 100 \% coton, manches longues, bleu marine ; été 2016

Sous Excel, ce fichier apparaîtra comme ceci (le point-virgule étant le séparateur de colonne) :

\begin{tabular}{|c|l|c|}
\hline Identifiant produit & \multicolumn{1}{|c|}{ Description produit } & Collection \\
\hline 102564 & Pull col V $-100 \%$ coton, manches longues, bleu ciel & été 2016 \\
\hline 102565 & Pull col V $-100 \%$ coton, manches longues, bleu marine & été 2016 \\
\hline
\end{tabular}

Dans ce scénario, la deuxième chaîne de description produit est une répétition à $88 \%$ de la première : I'outil de TAO fera bien une suggestion mais il faudra vérifier toute la chaîne. Dans l'analyse qui servira de base au devis, ceci n'apparaîtra même pas en tant que répétition ; il s'agira au mieux d'une correspondance partielle, avec une mémoire de traduction simulée de manière théorique.

En manipulant le fichier en revanche (soit avec Excel, soit directement lors de l'importation dans l'outil de TA0), on peut aisément obtenir une segmentation qui ressemblerait à ceci :

\begin{tabular}{|c|c|c|c|c|c|}
\hline ID produit & Description & Matériau & Complément & Couleur & Collection \\
\hline 102564 & Pull col V & $100 \%$ coton & manches longues & bleu ciel & été 2016 \\
\hline 102565 & Pull col V & $100 \%$ coton & manches longues & bleu marine & été 2016 \\
\hline
\end{tabular}

Pour obtenir ceci, la méthode la plus simple est de configurer l'outil de TAO pour qu'il considère le tiret et la virgule comme des séparateurs, ce qui prend quelques minutes. On obtient alors des segments plus courts et un volume de répétitions bien plus élevé, toutes les chaînes étant des répétitions parfaites sauf une (la couleur).

Au lieu de devoir éditer chaque produit, il suffira de traiter l'élément qui varie, et les répétitions auto-propagées par l'outil de TAO pourront être survolées très rapidement car le contexte ne les fait pas varier. On peut donc proposer un devis bien plus représentatif de l'effort de 
traduction réel, le but bien sûr étant de facturer un temps de travail qui a du sens et non un temps de travail maximal.

N'hésitez pas par ailleurs à prendre le temps d'analyser en détail le contenu des catalogues produits : au-delà de ces optimisations " de forme ", il s'avère parfois que des contenus périmés ou sans rapport aient été exportés par l'outil, et il est fréquent que le client n'ait même pas regardé le contenu du fichier (si, si). Dans un cas similaire à l'exemple proposé ci-dessus, le client a effectivement confirmé ne pas vouloir traduire les collections antérieures à 2017 (il fallait donc supprimer tout ce qui datait de 2016), un point que peu de concurrents avaient identifié.

\section{La question piège : le référencement}

Cette question est posée de manière récurrente par de nombreux clients, qui la plupart du temps n'ont pas vraiment d'idée de ce que cela représente hormis "être sur la première page de Google ". Nous parlerons ici du référencement naturel, aussi appelé SEO (Search Engine Optimisation - optimisation pour les moteurs de recherche), par opposition au référencement payant de type Adwords (les publicités que vous voyez sur la droite ou en haut des résultats sur Google).

Pour que Google propulse un site en première page, celui-ci doit répondre à une liste d'environ 200 critères, parmi lesquels figurent le choix de bons mots-clés et leur placement au bon endroit dans le site web. Mais si le site met trop de temps à charger des images, n'est mis à jour qu'une fois par an et/ou comporte moins de 10 pages, le travail sur les mots-clés ne servira strictement à rien.

Dans la plupart des cas, les clients n'ont pas de stratégie de référencement pour le site d'origine, mais attendent des miracles de la traduction. II vous suffira donc de leur demander habilement de vous faire part de leur stratégie pour la langue source en proposant de l'adapter pour la traduction. Dans $90 \%$ des cas, la discussion n'ira pas plus loin.

Dans l'éventualité où le client maîtriserait effectivement le référencement, je vous conseillerais alors de vous rapprocher d'une agence spécialisée, car il s'agit là d'un exercice très différent de la traduction. Pour la partie contenu, l'objet du référencement est d'identifier les mots-clés les plus recherchés par les internautes mais qui ne sont pas encore trop exploités par les concurrents, à l'aide d'outils spécialisés et souvent payants, afin de les insérer à des endroitsclés du site. Cela implique donc parfois de tout réécrire après avoir identifié les bons mots-clés, comme par exemple cette entreprise qui misait essentiellement sur son nom de marque pour son marché d'origine mais qui a dû s'y prendre totalement différemment pour la version internationale de son site, la marque étant parfaitement inconnue dans les pays ciblés. 
Le monde du web est fascinant et en perpétuelle évolution ; cet article sera sans doute à réécrire intégralement dans 5 ans. Mais en l'état, vous avez à présent toutes les cartes en main pour devenir l'expert rassurant que recherchent vos prospects!

aurelieduclos@itranslate.fr

\begin{abstract}
Aurélie Duclos est depuis toujours passionnée par l'anglais. Après une formation de documentaliste, métier qu'elle a exercé pendant 3 ans dans une école de commerce, elle a bifurqué par hasard vers la traduction en devenant chef de projet dans une petite agence, où elle a pu découvrir tout le circuit de la traduction. Pendant 5 ans, elle a eu en charge les projets et clients web auxquels son Master et son expérience précédente la destinaient naturellement, jusqu'à gérer une petite équipe dédiée à ces clients. Lorsque l'envie lui est venue de créer son activité de traductrice, le web est resté en bonne place : elle est aujourd'hui spécialisée dans la localisation d'interfaces et systèmes en ligne et traduit régulièrement des contenus webmarketing, des applications mobiles mais aussi tout ce qui relève du marketing et de la stratégie d'entreprise (de l'anglais vers le français bien entendu).
\end{abstract}

